\title{
The Association of Elective Hormone Therapy with Changes in Lipids Among Glucose Intolerant Postmenopausal Women in the Diabetes Prevention Program
}

\author{
Sherita H. Golden, M.D. M.H.S. ${ }^{1}$, Catherine Kim, M.D. M.P.H. ${ }^{2}$, Elizabeth Barrett-Connor, \\ M.D. ${ }^{3}$, Bin Nan, Ph.D. ${ }^{2}$, Shengchun Kong, M.S. ${ }^{2}$, Ronald Goldberg, M.D. ${ }^{4}$, and the Diabetes \\ Prevention Program Research Group \\ ${ }^{1}$ Johns Hopkins University, Baltimore, Maryland ${ }^{2}$ University of Michigan, Ann Arbor, Michigan \\ ${ }^{3}$ University of California, San Diego, San Diego, California ${ }^{4}$ University of Miami, Miami, Florida
}

\begin{abstract}
Objective-It is unclear how lipids change in response to lifestyle modification or metformin among postmenopausal glucose intolerant women using and not using hormone therapy (HT). We examined the one-year changes in lipids among postmenopausal, prediabetic women in the Diabetes Prevention Program (DPP), and whether changes were mediated by sex hormones.

Materials/Methods-We performed a secondary analysis of a randomized controlled trial of 342 women who used HT at baseline and year 1 and 382 women who did not use HT at either time point. Interventions included intensive lifestyle (ILS) with goals of weight reduction of at least 7\% of initial weight and 150 minutes per week of moderate intensity exercise, or metformin or placebo administered $850 \mathrm{mg}$ up to twice a day. Women were not randomized to HT. Main outcome measures were changes between baseline and study year 1 in low-density lipoprotein cholesterol (LDL-C), high-density lipoprotein cholesterol (HDL-C), and triglycerides.
\end{abstract}

Results-Compared to placebo, both ILS and metformin significantly reduced LDL-C and raised HDL-C among HT users, changes partially explained by change in estradiol and testosterone but independent of changes in waist circumference and 1/fasting insulin. In contrast, DPP interventions had no effect on LDL-C and HDL-C among non-HT users. ILS significantly lowered triglycerides among non-users but did not significantly change triglycerides among HT users. Metformin did not significantly change triglycerides among non-users but increased triglycerides among HT users.

Conclusions-The beneficial effects of ILS and metformin on lowering LDL-C and raising HDL-C differ depending upon concurrent HT use.

(C) 2013 Elsevier Inc. All rights reserved.

Address for Correspondence: Dr. Sherita Hill Golden, Johns Hopkins University School of Medicine, Division of Endocrinology and Metabolism, 1830 E. Monument Street, Suite 333, Baltimore, MD 21287 Tel: (410) 502-0993, Fax: (410) 955-8172, sahill@ jhmi.edu.

Publisher's Disclaimer: This is a PDF file of an unedited manuscript that has been accepted for publication. As a service to our customers we are providing this early version of the manuscript. The manuscript will undergo copyediting, typesetting, and review of the resulting proof before it is published in its final citable form. Please note that during the production process errors may be discovered which could affect the content, and all legal disclaimers that apply to the journal pertain.

Author Contributions

S.H.G., C.K., E.B.C., and R.G. designed and conducted the study; C.K., B.N. and S.K. collected and analyzed data; S.H.G., C.K., E.B.C., and R.G. interpreted the data; and S.H.G., C.K., E.B.C., and R.G. wrote and critically edited the manuscript.

Disclosure summary: S.H.G, C.K., E.B., B.N., and S.K. have no conflicts of interest to declare. R. G. previously received grant funding from Bristol Myers and Sanofi that ended in the past year. 


\section{Keywords}

sex hormones; lifestyle intervention; hormone therapy; lipids; women

Clinical trials of hormone therapy (HT) in postmenopausal women using oral estrogen with and without progesterone have shown that $\mathrm{HT}$ has both favorable and adverse effects on the lipid profile. While HT reduces LDL-cholesterol (LDL-C) and increases HDL-cholesterol (HDL-C) [1-10], HT also increases triglycerides [1-6] and does not prevent cardiovascular disease events $[2,4,5,11]$. HT has potentially complex effects on the lipid profile through modulation of serum sex hormone levels and effects on key enzymes in the lipid/lipoprotein metabolic pathway. Oral HT raises sex hormone binding globulin (SHBG), which may increase endogenous estradiol (E2) and decrease endogenous free testosterone (T) levels [12]. E2 may also impact lipoprotein levels via its binding to estrogen receptors in visceral and subcutaneous adipocytes $[13,14]$ and via its effects on lipoprotein lipase. Lipoprotein lipase activity is inversely correlated with E2 levels in obese women [15] and HT inhibits lipoprotein lipase activity, which can increase triglyceride levels [16]. E2 also inhibits hepatic lipase activity, thereby decreasing hydrolysis of cholesterol ester in HDL-C and increasing HDL-C levels [17]. These data suggest that interventions to manipulate serum sex hormones levels, beyond HT, may alter the lipid profile in post-menopausal women.

There are two potential ways to manipulate serum sex hormone levels--use of HT and lifestyle interventions including physical activity and dietary changes. Results of prior studies using lifestyle interventions have been mixed [18-20]. The Diabetes Prevention Program (DPP) was a randomized trial of intensive lifestyle change (ILS) and metformin in overweight, glucose-intolerant adults at high risk of diabetes. Both interventions had favorable effects on the lipid profiles including decreased triglycerides, increased HDL-C, and decreased small, dense LDL-C [21]. This analysis was not stratified by sex, menopausal status or HT use in women, although we have previously demonstrated that changes in serum sex hormones differ by HT use [22]. Due to the potential effects of exogenous estrogen on enzymes that affect lipid metabolism, the favorable effects of the DPP interventions on lipids could vary by HT use as both the interventions and HT use can alter serum sex hormone levels. We therefore sought to determine (1) the one year association of the DPP interventions on the lipid profile in glucose intolerant, obese post-menopausal women, (2) whether intervention-induced changes in lipid parameters were associated with changes in serum sex hormones, and (3) whether these changes differed by HT use status (Figure).

\section{Materials and Methods}

\section{Study Population}

Characteristics of DPP participants have been published [23]. Briefly, DPP inclusion criteria included age $\geq 25$ years, fasting plasma glucose (FPG) $95-125 \mathrm{mg} / \mathrm{dl}$ and 2-hour plasma glucose of 140-199 mg/dl following a 75-gram oral glucose load, age 225 years, and body mass index (BMI) $\geq 24 \mathrm{~kg} / \mathrm{m}^{2}$ ( $\geq 2 \mathrm{~kg} / \mathrm{m}^{2}$ for Asian-Americans). Participants were recruited from 26 clinical centers located throughout the United States (Baton Rouge, LA; Chicago, IL; Philadelphia, PA; Miami, FL; San Antonio, TX; Denver, CO; Boston, MA; Seattle, WA; Memphis, TN; Boston, MA; La Jolla, CA; New York, NY; Indianapolis, IN; Hyattsville, MD; Alhambra, CA; St. Louis, MO; Baltimore, MD; Albuquerque, NM; New York City, NY; Pittsburgh, PA; Honolulu, HI; and Phoenix, AZ). The Phoenix center is the site for 4 Indian Health Service areas and Chicago is the location for centers at the University of Chicago and Northwestern University. Written informed consent was obtained from all 
participants before screening, consistent with the guidelines of each participating center's institutional review board.

Eligible participants recruited between 1996 and 1999 were randomly assigned to one of three interventions: $850 \mathrm{mg}$ metformin twice daily, placebo twice daily, or ILS designed to achieve and maintain a weight reduction of at least $7 \%$ of initial body weight through consumption of a low-calorie, low-fat diet, and moderate physical activity for at least 150 $\mathrm{min} /$ week [23]. Weight and waist circumference were measured semiannually. Participants had an annual oral glucose tolerance test (OGTT) and semiannual FPG test. All women completed a baseline questionnaire about their menses, gynecological history including surgery, and exogenous hormone use (oral contraception or HT).

For this report, we included women who were postmenopausal at randomization. We excluded women enrolled at Native American centers, because they had not consented to participate in ancillary studies. Women were classified as being postmenopausal if they met any of the following criteria: bilateral oophorectomy, lack of menses for at least one year while retaining uterus and at least one ovary, cessation of menses prior to hysterectomy, cessation of menses within the past year and age $\geq 55$ years, and cessation of menses with hysterectomy and age $\geq 55$ years. Women classified as non-HT users did not use exogenous hormones at the time of randomization or 1-year follow-up.

There were 2191 women (excluding troglitazone users) enrolled into DPP at baseline. Of these 2191 women, 968 women reported that they had ceased menstruating due to bilateral oophorectomy, hysterectomy, or natural reasons. Of these 968 women, 384 women reported further that if they had ceased menstruating without surgery, it had been over a year since their last period and if they had ceased menstruating due to hysterectomy, they were over 55 years of age, and they did not use any time of estrogen at baseline or at follow-up. We excluded 2 women in the former group because they had very high estradiol levels for a total of 382 postmenopausal women who did not use any type of estrogen at baseline and followup (Figure). Of the postmenopausal women, 324 also reported oral estrogen at both baseline and 1 year follow-up (Figure). The remaining women took another form of estrogen at baseline or at follow-up.

\section{Assessment of lipids [21]}

Blood was drawn after a requested 12-h fast at baseline and annually thereafter. Total plasma cholesterol and triglyceride levels were measured using enzymatic methods standardized to the Centers for Disease Control and Prevention reference methods [24]. HDL fractions of cholesterol were measured using dextran sulfate- $\mathrm{Mg}^{2+}$ to precipitate all of the apolipoprotein B (apoB)-containing lipoproteins [25]. LDL-C was calculated by the Friedewald equation [26] unless the triglyceride level was $>4.5 \mathrm{mmol} / \mathrm{L}$, in which case lipoprotein fractions were separated by preparative ultracentrifugation [27] and LDL subfraction was determined by density gradient ultracentrifugation.

\section{Assessment of endogenous sex hormones and sex hormone binding globulin (SHBG)}

SHBG, follicle stimulating hormone (FSH), total E2, total T, and dehydroepiandrosterone (DHEA) were measured at Endoceutics (Quebec City, Canada) on heparinized plasma collected at baseline and year 1. SHBG was measured using ELISA (Bioline) with interassay coefficients of variation of 7.8 and 5.0 at 18.2 and $63.1 \mathrm{nmol} / \mathrm{l}$, respectively. FSH was measured using ELISA (Bioline) with interassay coefficients of variation of 3.6 and 4.4 at 27.1 and $72.9 \mathrm{mIU} / \mathrm{ml}$, respectively. E2, T, and DHEA were analyzed using gas chromatography/mass spectrometry at Endoceutics As previously described [28], the gas chromatography/mass spectrometry system uses a 50\% phenyl-di-methyl polysiloxane 
capillary column ( $30 \mathrm{~m} \times 0.25 \mathrm{~mm}$ internal diameter, $0.15 \mu \mathrm{m}$ film thickness) with helium as the carrier gas. Analytes were detected using a HP5973 quadruple mass spectrometer equipped with a chemical ionization source. The limits of detection were $3.0 \mathrm{pg} / \mathrm{ml}$ for total $\mathrm{E} 2 ; 8.0 \mathrm{ng} / \mathrm{dl}$ for total $\mathrm{T}$, and $0.30 \mathrm{ng} / \mathrm{ml}$ for DHEA. Interassay coefficients of variation for E2 were $17.5 \%$ at $4.7 \mathrm{pg} / \mathrm{ml}$, for T were $13.0 \%$ at $14 \mathrm{ng} / \mathrm{dl}$, and for DHEA were $24.0 \%$ at $0.77 \mathrm{ng} / \mathrm{ml}$. Bioavailable and free T and E2 were calculated according to the method described by Sodergard and colleagues (macro courtesy of Frank Stanczyk, University of Southern California) taking the concentrations of total T, total E2, and SHBG into account and assuming a fixed albumin concentration of $4.0 \mathrm{~g} / \mathrm{dl}$ [29].

As customary in most epidemiologic studies, hormone levels were assessed based on single samples at baseline and one year. Single measurements of T and SHBG reliably characterize average levels over a 2 to $3 \mathrm{yr}$ period; E2 levels are less reproducible and more subject to intra-individual variation [30,31]. Endogenous sex hormone measurements have been shown to be stable for $>10$ years in frozen plasma stored at $-70^{\circ} \mathrm{C}$ [32], as was true of these DPP samples.

\section{Assessment of covariates}

Participants brought all prescription medicines, including cholesterol-lowering medications and estrogens, to each quarterly clinic visit, and all were recorded. Height was measured on a stadiometer to the nearest $0.5 \mathrm{~cm}$, body weight was measured on a calibrated balance scale to the nearest $0.1 \mathrm{~kg}$, and BMI $\left(\mathrm{kg} / \mathrm{m}^{2}\right)$ was computed from body weight and height. Waist was defined as the midpoint between the highest point of the iliac crest and the lowest point of the costal margin at the midaxillary line, and waist circumference was measured with a cloth tape [33]. Each measurement was recorded twice during the baseline screening visit and averaged. A third measurement was taken if the variability was greater than a predefined value. Staff members performing these measurements were certified annually. Plasma insulin was measured fasting as previously described [34]. Insulin sensitivity was assessed using inverse fasting insulin levels (1/fasting insulin) [34].

\section{Statistical Analysis}

All analyses were stratified by no estrogen therapy use at baseline or 1-year follow-up vs. oral estrogen therapy with or without progestogen at baseline and year 1 follow-up. Baseline characteristics were described using percentages for categorical variables and means (SD) for quantitative variables. For variables where the distribution was skewed, log-transformed values and median values were used as indicated in Table 1. In order to assess the association between randomization assignment and change in each cholesterol sub-fraction between baseline and year 1 follow-up, as well as change in body mass measures and sex hormone measures, we used t-tests to compare levels of change between randomization arms. Change was calculated as year 1 level minus baseline level.

To determine if any intervention effects upon change in lipid sub-fraction were confounded by age, race/ethnicity, or cholesterol-lowering medication use, we constructed linear regression models which adjusted for these factors. The correlation between FSH and age was 0.29 , and baseline follicle stimulating hormone (FSH) levels differed between women randomized to metformin and other arms, so both measures were included in the models. To determine if the observed changes in sex hormones were associated with changes in lipid fraction, we further adjusted for change in sex hormone level and then for change in waist circumference and insulin sensitivity. The SAS analysis system was used for all analyses (SAS Institute, Cary, NC). 


\section{Results}

\section{Baseline Characteristics}

As shown in Table 1, baseline participant characteristics did not differ by randomization arms. Postmenopausal women not using HT had a median age of 58.5 years and interquartile range of 52.8 to 64.3 years; approximately half were Caucasian, a quarter AfricanAmerican, and about $16 \%$ were Hispanic. The majority of women reported a natural menopause without or prior to gynecologic surgery, with an average of 15 years since their last menstrual period (median 12.9 years and interquartile range 6.5 to 21.0 years).

Post-menopausal women using HT had a median age of 56.5 years and interquartile range of 52.0 to 61.0 years; about two-thirds were Caucasian, $16 \%$ African-American, and $13 \%$ were Hispanic. A similar proportion of women reported natural and surgical menopause, with an average of 14 years since their last menstrual period (median 12.1 years and interquartile range 5.9 to 20.9 years). Of the 324 HT users, 266 women were estrogen-only users at baseline and 58 women used estrogen-progestin; at year 1 follow-up, 258 women were estrogen-only users and 66 women used estrogen-progestin. The most common estrogens reported at baseline and follow-up were oral conjugated equine estrogen (Premarin), followed by 17-beta-estradiol (Estrace), followed by esterified estrogen (Estratab, Menest) and estropipate (Ogen, Ortho-Est). Of the 211 women who had a hysterectomy, 8 (4\%) were using a progestin at baseline and $7(3 \%)$ were using a progestin at follow-up. Of the 113 women who did not report a hysterectomy, $44 \%$ were using a progestin at baseline and $52 \%$ were using a progestin at follow-up.

In comparing the two groups, non-HT users were older, less likely to be Caucasian, more likely to have experienced natural menopause and less likely to have had a hysterectomy than HT users. Years since the final menstrual period were similar between the two groups. Reflecting the enrollment criteria for the DPP, HT users and non-users were overweight at baseline, with non-HT users having higher weight, waist circumference, and BMI than nonHT users; however, both groups had reductions in weight, waist circumference, and fasting insulin during the first year of the study. HT users had higher E2 and SHBG and lower FSH and DHEA than non-HT users and HT users had a significantly greater rise in SHBG over 1 year compared to non-HT users. Women using HT at baseline had lower LDL-C and higher HDL-C and triglycerides than women not using HT.

We determined whether associations differed by estrogen-only versus estrogen + progestogen therapy; however, since there was not significant statistical interactions by type of HT, combined analyses are presented as the primary results.

\section{Association between interventions and change in LDL (Table 2)}

Compared to placebo, both ILS and metformin significantly reduced LDL among HT users after adjustment for age, race/ethnicity, cholesterol medication use, and FSH (Table 2, base model). This effect was most pronounced among the estrogen-only users (data not shown). Interventions did not significantly change LDL among non-HT users.

SHBG and DHEA-The association of ILS and metformin among HT users with change in LDL persisted following adjustment for change in SHBG, DHEA, waist circumference, and $1 /$ fasting insulin.

Total E2-The association of ILS and metformin with change in LDL was attenuated after adjustment for change in total E2. Total E2 declined with the interventions among HT and 
estrogen-only users (latter data not shown). The associations became significant again following the addition of change in waist circumference and $1 /$ fasting insulin to the models.

Total T-The association of ILS and metformin interventions with change in LDL-C was attenuated following adjustment for change in total T, which declined among HT users but not among estrogen-only users (latter data not shown). Associations became significant again following the addition of change in waist circumference and 1/fasting insulin to the models.

\section{Association between interventions and change in HDL (Table 3)}

Compared to placebo, both ILS and metformin significantly increased HDL among HT users (Table 3, base model). Interventions did not significantly change HDL among non-HT users or estrogen-only users.

SHBG-The association of ILS and metformin with change in HDL-C among HT users persisted following adjustment for change in SHBG; however, it was attenuated following adjustment for change in waist circumference and 1/fasting insulin.

DHEA-The association of ILS and metformin with change in HDL-C among HT users persisted following adjustment for change in DHEA but was attenuated following multivariable adjustment, including change in DHEA, in the metformin arm.

Total E2-The associations of the interventions with change in HDL-C among HT users were attenuated in ILS following adjustment for change in total E2, change in waist circumference and $1 /$ fasting insulin, but persisted in metformin users.

Total T-The associations of the interventions with change in HDL-C among HT users was attenuated following adjustment for change in total T in the ILS arm but not the metformin arm. Further adjustment for change in waist circumference and 1 /fasting insulin attenuated the association in the metformin arm.

\section{Association between interventions and change in triglycerides (Table 4)}

Compared to placebo, the ILS but not metformin arm was associated with a significant decrease in triglycerides only among non-HT users. This association was not seen among HT and estrogen-only users (Table 4, base model). The association of ILS with decrease in triglycerides among non-HT users persisted following adjustment for change in all sex hormones (SHBG, DHEA, total E2, and total T), waist circumference, and 1/fasting insulin.

The metformin intervention was associated with an increase in triglycerides among HT and estrogen-only users following adjustment for change in SHBG and this persisted following additional multivariable adjustment. The association of the metformin intervention with increases in triglycerides among HT users persisted following adjustment for change in other hormones, including DHEA, total E2, and total T.

Subsidiary Analyses-Models substituting the homeostasis model assessment of insulin resistance (HOMA-IR) for 1/fasting insulin had similar results (data not shown). Models without FSH levels had similar results (data not shown).

\section{Discussion}

Our study shows that there are differential effects of ILS and metformin on lipoprotein measures in glucose-intolerant overweight post-menopausal women who were or were not 
taking HT. LDL-C and HDL-C were generally favorably impacted by ILS and metformin in HT users, which were partially explained by change in E2 and T, but was independent of changes in waist circumference and $1 /$ fasting insulin. In contrast, interventions had no significant impact on LDL-C and HDL-C among non-HT users. The effect of ILS and metformin on triglyceride levels also differed depending on HT status. Among non-HT users, ILS significantly lowered triglycerides; however, among HT users, including estrogen-only users, ILS and metformin were associated with increased triglycerides. Thus HT use appears to influence the lipid responses to ILs and metformin in directions that mimic both the favorable (lower LDL-C, higher HDL-C) as well as unfavorable (increase in triglycerides) effects of HT on lipid and lipoprotein levels.

To our knowledge, previous studies have not evaluated the impact of ILS and metformin by HT use, despite the potential effects of such interventions on sex hormones. The basis for the lipoprotein response to ILS is complex, reflecting effects of weight reduction, reduced saturated fat intake, and increased physical activity on lipoprotein production and clearance. In prior DPP analyses, the major effect of ILS and metformin appears to be reduced VLDL production leading to a fall in triglycerides levels [21]. The fall in triglycerides together with changes in HDL-C and LDL-C remodeling and clearance results in a fall in LDL-C and an increase in HDL-C. Physical exercise also has a favorable effect on serum lipids, particularly on lowering of triglycerides by promoting catabolism of triglyceride-rich lipoproteins [35,36]. Although metformin was not associated with a change in triglyceride level overall in the DPP, others have shown a modest effect of metformin to lower triglycerides levels, possibly as a result of an increase in lipoprotein lipase activity through activation of 5' adenosine monophosphate-activated protein kinase (AMPK) activity in skeletal muscle in vitro [37] and adipose tissue in vivo [38]. The effect of metformin and exercise to increase AMPK also leads to an elevation in adipose tissue lipolysis with free fatty acids directed into oxidative pathways and away from triglyceride and cholesterol synthesis. Metformin also leads to an overall modest reduction in LDL-C and increase in HDL-C, as others have shown, the mechanisms of which are not known [38].

Surprisingly, we found that HT use appeared to antagonize the beneficial effect of ILS and metformin on triglyceride levels, with ILS having minimal effect on triglyceride levels and metformin being associated with increased triglyceride levels. As reviewed earlier, exogenous E2 inhibits lipoprotein lipase activity, which can increase triglyceride levels [16]. In addition, E2 increases VLDL production [39]. In our population, the effect of E2 appeared to override the effects of the interventions on triglyceride concentrations, particularly in the metformin group. This is consistent with the general observation that ILS lowered triglyceride more than did metformin in the DPP [21]. Furthermore, these findings suggest that ILS ameliorates the triglyceride-raising effect of estrogen whereas metformin treatment did not. This effect is different than that seen in non-obese women with polycystic ovary syndrome, where the use of an oral contraceptive with ethinyl E2 in combination with metformin raised HDL-C in one study [40] and did not have an adverse effect on triglycerides or other lipid parameters in two studies [40,41]. Further studies are needed to shed light on the reason that HT therapy appears to antagonize the effect of ILS and metformin effects on triglyceride levels.

HT use had the anticipated favorable effect in lowering LDL-C and raising HDL-C, which appeared to be enhanced further by ILS and metformin interventions. The LDL-lowering effects of ILS and metformin were partially explained by change in E2 and T levels. The HDL-raising effect of ILS was also partially explained by change in E2 and T; however, change in sex hormones did not explain the HDL-raising effect in the metformin arm. E2 inhibits hepatic lipase activity, decreasing hydrolysis of cholesterol ester in HDL-C and resulting in higher HDLC levels [17], and increases the concentration of apo AI-containing 
HDL-C particles [42]. In our models, adjustment for change in waist circumference and insulin resistance explained the HDLC raising effect of ILS and metformin. This is not surprising given the association of insulin resistance and central adiposity with HDL-C levels [43].

Oral E2 also accelerates LDL-C catabolism and in animal models, has resulted in an increase in hepatic LDL receptors, which would accelerate LDL-C removal from the circulation [42]. In human studies, $17 \beta$-estradiol has increased the fractional catabolic rate of LDL-C and apoB, even though the LDL-C production rate was not decreased [44]. The favorable effect of HT on LDL-C may also be mediated through activation of liver AMPK by E2, as suggested by animal studies [45]. Once activated, AMPK phosphorylates and inactivates acetyl-CoA carboxylase and 3-hydroxy-3-methylglutaryl-CoA reductase, inhibiting de novo fatty acid and cholesterol synthesis [46], which would result in lower LDL-C. We hypothesize that the AMPK activating effects of metformin and exercise in the ILS group may have a synergistic effect with E2, contributing further to the favorable lipid effects. Future laboratory-based studies are needed to explore these mechanisms further.

The strengths of our study include the randomized design, rigorous interventions that impacted metabolic parameters in a statistical and clinical meaningful manner, measurement of several lipid parameters, the ability to specifically examine the effect of HT and is interaction with our interventions, and assessment of several potential explanatory and/or confounding variables, including cholesterol medication use and change in sex hormones, 1/ fasting insulin, and waist circumference. Our population also included women if multiple race/ethnicities, increasing the generalizability of our findings.

Several limitations should be kept in mind in interpreting our data. First, residual confounding is possible as we used a proxy of insulin resistance and not a direct measure. Second, we performed a secondary analysis of a randomized trial which was not designed a priori to specifically assess the impact of HT on lipid parameters by treatment assignment; although, favorable effects of the interventions on the lipid profile were observed for this cohort [21]. Third, we did not have data on hormone doses to include in our analyses. Fourth, our results may only be applicable to obese, glucose intolerant women as our cohorts did not recruit lean women. Finally, we do not have data that allow us to assess the molecular mechanisms through which HT acts synergistically with metformin and ILS to raise HDL-C and lower LDLC and through which HT antagonizes the effect of metformin and ILS on triglycerides.

Our findings suggest that the beneficial effects of ILS and metformin on lowering LDL-C and raising HDL-C are enhanced by HT use in glucose intolerant postmenopausal women, as these effects were not observed in women not taking HT. It is unknown whether these favorable lipid changes in HT users will translate into favorable clinical cardiovascular outcomes for the subpopulation of glucose intolerant women since HT has not been beneficial in reducing cardiovascular events in women as a whole. The reason for the apparent antagonistic effect of HT on the triglyceride-lowering effect of ILS and metformin is unknown and future studies are needed to explore the molecular mechanisms contributing to this effect. The disparate effect of HT on LDL-C and HDL-C compared to triglycerides response to ILS and metformin might contribute to the failure to date of HT trials to demonstrate beneficial cardiovascular disease outcomes. However, the basis for the unfavorable effects of an elevation in triglyceride levels may at least in part be related to the frequent presence of low HDL-C. It is therefore possible that the long-term cardiovascular risk due the estrogen-associated rise in triglyceride levels is offset by the concomitant fall in LDL-C and increase in HDL-C. 


\section{Supplementary Material}

Refer to Web version on PubMed Central for supplementary material.

\section{Acknowledgments}

The Investigators gratefully acknowledge the commitment and dedication of the participants of the DPP. The opinions expressed are those of the investigators and do not necessarily reflect the views of the Indian Health Service or other funding agencies. A complete list of centers, investigators, and staff can be found in the supplemental appendix.

Funding The project described was supported by Award Numbers U01DK048489, R01DK083297, and K23DK071552 from The National Institute of Diabetes and Digestive and Kidney Diseases (NIDDK) of the National Institutes of Health. The NIDDK provided funding to the clinical centers and the Coordinating Center for the design and conduct of the study; collection, management, analysis, and interpretation of the Diabetes Prevention Program. The Southwestern American Indian Centers were supported directly by the NIDDK and the Indian Health Service. The General Clinical Research Center Program, National Center for Research Resources supported data collection at many of the clinical centers. Funding for data collection and participant support was also provided by the National Institute of Child Health and Human Development, the National Institute on Aging, the Office of Research on Women's Health, the Office of Research on Minority Health, the Centers for Disease Control and Prevention, and the American Diabetes Association. Bristol-Myers Squibb and Parke-Davis provided medication. This research was also supported, in part, by the intramural research program of the NIDDK. LifeScan Inc., Health O Meter, Hoechst Marion Roussel, Inc., Merck-Medco Managed Care, Inc., Merck and Co., Nike Sports Marketing, Slim Fast Foods Co., and Quaker Oats Co. donated materials, equipment, or medicines for concomitant conditions. McKesson BioServices Corp., Matthews Media Group, Inc., and the Henry M. Jackson Foundation provided support services under subcontract with the Coordinating Center. The opinions expressed are those of the investigators and do not necessarily reflect the views of the Indian Health Service or other funding agencies.

\section{Abbreviations}

\begin{tabular}{ll} 
AMPK & \multicolumn{1}{l}{ ' adenosine monophosphate-activated protein kinase } \\
apoB & apolipoprotein B \\
BMI & body mass index \\
DPP & Diabetes Prevention Program \\
DHEA & dehydroepiandrosterone \\
E2 & estradiol \\
FPG & fasting plasma glucose \\
FHS & follicle stimulating hormone \\
HDL-C & high density lipoprotein cholesterol \\
HOMA-IR & homeostatic model assessment of insulin resistance \\
HT & hormone therapy \\
ILS & intensive lifestyle therapy \\
LDL-C & low density lipoprotein cholesterol \\
OGTT & oral glucose tolerance test \\
SD & standard deviation \\
SHBG & sex hormone binding globulin \\
T & testosterone \\
VLDL & very low density lipoprotein \\
\end{tabular}




\section{Reference List}

1. The Writing Group for the PEPI Trial. Effects of estrogen or estrogen/progestin regimens on heart disease risk factors in postmenopausal women. The Postmenopausal Estrogen/Progestin Interventions (PEPI) Trial. The Writing Group for the PEPI Trial [see comments] [published erratum appears in JAMA 1995 Dec 6;274(21):1676]. JAMA. 1995; 273:199-208. [PubMed: 7807658]

2. Hulley S, Grady D, Bush T, et al. Randomized trial of estrogen plus progestin for secondary prevention of coronary heart disease in postmenopausal women. Heart and Estrogen/progestin Replacement Study (HERS) Research Group [see comments]. JAMA. 1998; 280:605-613. [PubMed: 9718051]

3. Writing Group for the Women's Health Initiative Investigators. Risks and benefits of estrogen plus progestin in healthy postmenopausal women: principal results from the Women's Health Initiative randomized controlled trial. JAMA. 2002; 288:321-333. [PubMed: 12117397]

4. Manson JE, Hsia J, Johnson KC, et al. Estrogen plus progestin and the risk of coronary heart disease. N.Engl.J.Med. 2003; 349:523-534. [PubMed: 12904517]

5. Anderson GL, Limacher M, Assaf AR, et al. Effects of conjugated equine estrogen in postmenopausal women with hysterectomy: the Women's Health Initiative randomized controlled trial. JAMA. 2004; 291:1701-1712. [PubMed: 15082697]

6. Waters DD, Alderman EL, Hsia J, et al. Effects of hormone replacement therapy and antioxidant vitamin supplements on coronary atherosclerosis in postmenopausal women: a randomized controlled trial. JAMA. 2002; 288:2432-2440. [PubMed: 12435256]

7. Herrington DM, Reboussin DM, Brosnihan KB, et al. Effects of estrogen replacement on the progression of coronary-artery atherosclerosis. N.Engl.J.Med. 2000; 343:522-529. [PubMed: 10954759]

8. Manolio TA, Furberg CD, Shemanski L, et al. Associations of postmenopausal estrogen use with cardiovascular disease and its risk factors in older women. The CHS Collaborative Research Group. Circulation. 1993; 88:2163-2171. [PubMed: 8222111]

9. Nabulsi AA, Folsom AR, White A, et al. Association of hormone-replacement therapy with various cardiovascular risk factors in postmenopausal women. The Atherosclerosis Risk in Communities Study Investigators [see comments]. N.Engl.J.Med. 1993; 328:1069-1075. [PubMed: 8384316]

10. Vaziri SM, Evans JC, Larson MG, et al. The impact of female hormone usage on the lipid profile. The Framingham Offspring Study [see comments]. Arch Intern Med. 1993; 153:2200-2206. [PubMed: 8215723]

11. Rossouw JE, Anderson GL, Prentice RL, et al. Risks and benefits of estrogen plus progestin in healthy postmenopausal women: principal results From the Women's Health Initiative randomized controlled trial. JAMA. 2002; 288:321-333. [PubMed: 12117397]

12. Rosner W. Plasma steroid-binding proteins. Endocrinol.Metab Clin.North Am. 1991; 20:697-720. [PubMed: 1778174]

13. Pedersen SB, Hansen PS, Lund S, et al. Identification of oestrogen receptors and oestrogen receptor mRNA in human adipose tissue. Eur.J.Clin.Invest. 1996; 26:262-269. [PubMed: 8732482]

14. Pedersen SB, Fuglsig S, Sjogren P, et al. Identification of steroid receptors in human adipose tissue. Eur.J.Clin.Invest. 1996; 26:1051-1056. [PubMed: 9013078]

15. Iverius $P$, Brunzell JD. Relationship between lipoprotein lipase activity and plasma sex steroid levels in obese women. Journal of Clinical Investigation. 1988; 82:1106-1112. [PubMed: 3417867]

16. Godsland IF. Effects of postmenopausal hormone replacement therapy on lipid, lipoprotein, and apolipoprotein (a) concentrations: analysis of studies published from 1974-2000. Fertil.Steril. 2001; 75:898-915. [PubMed: 11334901]

17. Serougne C, Feurgard C, Hajri T, et al. Catabolism of HDL1 cholesteryl ester in the rat. Effect of ethinyl estradiol treatment. C.R.Acad.Sci.III. 1999; 322:591-596. [PubMed: 10488433] 
18. Friedenreich CM, Woolcott CG, McTiernan A, et al. Alberta physical activity and breast cancer prevention trial: sex hormone changes in a year-long exercise intervention among postmenopausal women. J.Clin.Oncol. 2010; 28:1458-1466. [PubMed: 20159820]

19. McTiernan A, Tworoger SS, Rajan KB, et al. Effect of exercise on serum androgens in postmenopausal women: a 12-month randomized clinical trial. Cancer Epidemiol.Biomarkers Prev. 2004; 13:1099-1105. [PubMed: 15247119]

20. McTiernan A, Tworoger SS, Ulrich CM, et al. Effect of exercise on serum estrogens in postmenopausal women: a 12-month randomized clinical trial. Cancer Res. 2004; 64:2923-2928. [PubMed: 15087413]

21. Ratner R, Goldberg R, Haffner S, et al. Impact of intensive lifestyle and metformin therapy on cardiovascular disease risk factors in the diabetes prevention program. Diabetes Care. 2005; 28:888-894. [PubMed: 15793191]

22. Kim C, Kong S, Laughlin GA, et al. Reduction in Glucose Among Postmenopausal Women Who Use and Do Not Use Estrogen Therapy. Menopause. (in press) epub Nov 192012.

23. Knowler WC, Barrett-Connor E, Fowler SE, et al. Reduction in the incidence of type 2 diabetes with lifestyle intervention or metformin. N.Engl.J Med. 2002; 346:393-403. [PubMed: 11832527]

24. Warnick GR. Enzymatic methods for quantification of lipoprotein lipids. Methods Enzymol. 1986; 129:101-123. [PubMed: 3724535]

25. Warnick GR, Benderson J, Albers JJ. Dextran sulfate-Mg2+ precipitation procedure for quantitation of high-density-lipoprotein cholesterol. Clin.Chem. 1982; 28:1379-1388. [PubMed: 7074948]

26. Friedewald WT, Levy RI, Fredrickson DS. Estimation of the concentration of low-density lipoprotein cholesterol in plasma, without use of the preparative ultracentrifuge. Clin.Chem. 1972; 18:499-502. [PubMed: 4337382]

27. U.S Department of Health and Human Services. Lipid Research Clinics Program, Lipid and Lipoprotein Analysis, Manual of Laboratory Operations. National Institutes of Health; Bethesda, MD: 1983.

28. Kim C, Kong S, Laughlin GA, et al. Endogenous Sex Hormone Changes in Postmenopausal Women in the Diabetes Prevention Program. J.Clin.Endocrinol.Metab. 2012; 97:2853-2861. [PubMed: 22689695]

29. Sodergard R, Backstrom T, Shanbhag V, et al. Calculation of free and bound fractions of testosterone and estradiol-17 beta to human plasma proteins at body temperature. J Steroid Biochem. 1982; 16:801-810. [PubMed: 7202083]

30. Hankinson SE, Manson JE, Spiegelman D, et al. Reproducibility of plasma hormone levels in postmenopausal women over a 2-3-year period. Cancer Epidemiol.Biomarkers Prev. 1995; 4:649654. [PubMed: 8547832]

31. Bolelli G, Muti P, Micheli A, et al. Validity for epidemiological studies of long-term cryoconservation of steroid and protein hormones in serum and plasma. Cancer Epidemiol.Biomarkers Prev. 1995; 4:509-513. [PubMed: 7549807]

32. Laughlin GA, Barrett-Connor E, May S. Sex-specific association of the androgen to oestrogen ratio with adipocytokine levels in older adults: the Rancho Bernardo Study. Clin.Endocrinol.(Oxf). 2006; 65:506-513. [PubMed: 16984244]

33. Fujimoto WY, Jablonski KA, Bray GA, et al. Body size and shape changes and the risk of diabetes in the diabetes prevention program. Diabetes. 2007; 56:1680-1685. [PubMed: 17363740]

34. Kitabchi AE, Temprosa M, Knowler WC, et al. Role of insulin secretion and sensitivity in the evolution of type 2 diabetes in the diabetes prevention program: effects of lifestyle intervention and metformin. Diabetes. 2005; 54:2404-2414. [PubMed: 16046308]

35. Frosig C, Jorgensen SB, Hardie DG, et al. $5^{\prime}$-AMP-activated protein kinase activity and protein expression are regulated by endurance training in human skeletal muscle. Am.J.Physiol Endocrinol.Metab. 2004; 286:E411-E417. [PubMed: 14613924]

36. Takekoshi K, Fukuhara M, Quin Z, et al. Long-term exercise stimulates adenosine monophosphate-activated protein kinase activity and subunit expression in rat visceral adipose tissue and liver. Metabolism. 2006; 55:1122-1128. [PubMed: 16839850] 
37. Ohira M, Miyashita Y, Murano T, et al. Metformin promotes induction of lipoprotein lipase in skeletal muscle through activation of adenosine monophosphate-activated protein kinase. Metabolism. 2009; 58:1408-1414. [PubMed: 19570550]

38. Boyle JG, Logan PJ, Jones GC, et al. AMP-activated protein kinase is activated in adipose tissue of individuals with type 2 diabetes treated with metformin: a randomised glycaemia-controlled crossover study. Diabetologia. 2011; 54:1799-1809. [PubMed: 21455728]

39. Schaefer EJ, Foster DM, Zech LA, et al. The effects of estrogen administration on plasma lipoprotein metabolism in premenopausal females. J.Clin.Endocrinol.Metab. 1983; 57:262-267. [PubMed: 6408108]

40. Fruzzetti F, Perini D, Lazzarini V, et al. Comparison of effects of $3 \mathrm{mg}$ drospirenone plus $20 \mathrm{mug}$ ethinyl estradiol alone or combined with metformin or cyproterone acetate on classic metabolic cardiovascular risk factors in nonobese women with polycystic ovary syndrome. Fertil.Steril. 2010; 94:1793-1798. [PubMed: 19931080]

41. Elter K, Imir G, Durmusoglu F. Clinical, endocrine and metabolic effects of metformin added to ethinyl estradiol-cyproterone acetate in non-obese women with polycystic ovarian syndrome: a randomized controlled study. Hum.Reprod. 2002; 17:1729-1737. [PubMed: 12093831]

42. Walsh BW, Schiff I, Rosner B, et al. Effects of postmenopausal estrogen replacement on the concentrations and metabolism of plasma lipoproteins. N.Engl.J.Med. 1991; 325:1196-1204. [PubMed: 1922206]

43. Bonora E, Kiechl S, Willeit J, et al. Prevalence of insulin resistance in metabolic disorders: the Bruneck Study. Diabetes. 1998; 47:1643-1649. [PubMed: 9753305]

44. Campos H, Walsh BW, Judge H, et al. Effect of estrogen on very low density lipoprotein and low density lipoprotein subclass metabolism in postmenopausal women. J.Clin.Endocrinol.Metab. 1997; 82:3955-3963. [PubMed: 9398695]

45. Trapani L, Violo F, Pallottini V. Hypercholesterolemia and 3-hydroxy-3-methylglutaryl coenzyme A reductase regulation in aged female rats. Exp.Gerontol. 2010; 45:119-128. [PubMed: 19895880]

46. Zhang BB, Zhou G, Li C. AMPK: an emerging drug target for diabetes and the metabolic syndrome. Cell Metab. 2009; 9:407-416. [PubMed: 19416711] 


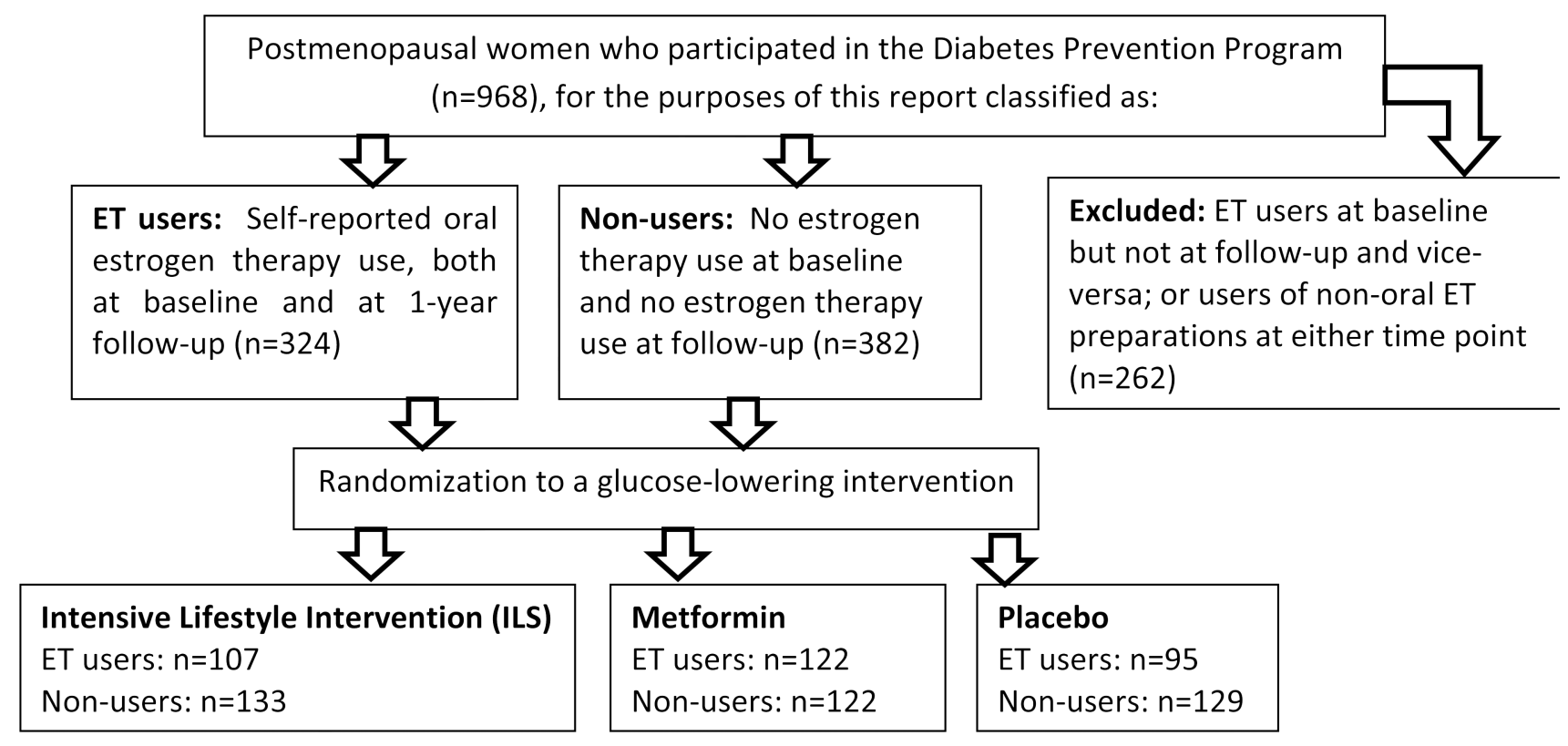

Figure 1.

Secondary analysis design. We conducted an analysis of the effectiveness of interventions in a randomized trial (the Diabetes Prevention Program), among postmenopausal women who were either 1) oral estrogen users at baseline and at 1-year follow-up or 2) non-estrogen users at baseline and at 1-year follow-up.

Adapted from: Kim C et al. Reduction in Glucose Among Postmenopausal Women Who Use and Do Not Use Estrogen Therapy. Menopause, 2012, In press. 
Table 1

Baseline characteristics of participants by hormone therapy (HT) use status. Means (standard deviation) or percentages shown unless otherwise indicated.

\begin{tabular}{|c|c|c|c|}
\hline Characteristic & $\begin{array}{l}\text { Not Using Hormone Therapy } \\
\text { N=382 }\end{array}$ & $\begin{array}{l}\text { Using Oral Hormone Therapy at } \\
\text { Baseline N=324 }\end{array}$ & P-value * \\
\hline Age (years) & $59(9)$ & $57(8)$ & $<0.001$ \\
\hline Race/ethnicity (\%) & & & $<0.01$ \\
\hline Caucasian & 53 & 66 & \\
\hline African-American & 28 & 16 & \\
\hline Hispanic & 16 & 13 & \\
\hline Asian & & 4 & \\
\hline Type of menopause (\%) & & & $<0.0001$ \\
\hline Bilateral oophorectomy & 19 & 42 & \\
\hline Natural menopause & 67 & 39 & \\
\hline Age 255 years and hysterectomy & 13 & 19 & \\
\hline Hysterectomy (\%) & 37 & 65 & $<0.0001$ \\
\hline Years since final menstrual period & $15(10)$ & $14(9)$ & 0.34 \\
\hline Cholesterol medication at baseline (\%) & 5 & 7 & 0.38 \\
\hline Cholesterol medication at follow-up (\%) & 10 & 10 & 0.90 \\
\hline Baseline weight (kg) & $91(19.7)$ & $87.6(17.8)$ & 0.038 \\
\hline Change in weight $(\mathrm{kg})$ & $-3.6(5.6)$ & $-3.4(5.6)$ & 0.69 \\
\hline Baseline waist circumference $(\mathrm{cm})$ & $104(14)$ & $101(14)$ & $<0.01$ \\
\hline Change in waist circumference $(\mathrm{cm})$ & $-4(6)$ & $-3(7)$ & 0.12 \\
\hline Baseline BMI $\left(\mathrm{kg} / \mathrm{m}^{2}\right)$ & $34.6(6.8)$ & $33.3(6.5)$ & 0.013 \\
\hline Change in BMI $\left(\mathrm{kg} / \mathrm{m}^{2}\right)$ & $-1.4(2.2)$ & $-1.3(2.1)$ & 0.57 \\
\hline Baseline fasting insulin (IU/l) & $26.4(16)$ & $23.4(12.1)$ & $<0.01$ \\
\hline Change in fasting insulin (IU/l) & $-3.5(14.2)$ & $-0.7(17.4)$ & 0.48 \\
\hline Follicle Stimulating Hormone (IU/l) & $55.3(26.6)$ & $34.8(22.5)$ & $\mid<0.0001$ \\
\hline Change in FSH & $3.1(13.4)$ & $0.6(15.4)$ & 0.51 \\
\hline Baseline SHBG (nM/l), median (IQR) & $33.2(18.3)$ & $85.3(77.0)$ & $<0.0001$ \\
\hline Change in SHBG (nM/l) & $4.2(17.8)$ & $13.1(43.3)$ & 0.0002 \\
\hline Baseline DHEA (ng/ml), median (IQR) & $1.6(1.3)$ & $1.3(1.2)$ & 0.01 \\
\hline
\end{tabular}




\begin{tabular}{|c|c|c|c|}
\hline Characteristic & $\begin{array}{l}\text { Not Using Hormone Therapy } \\
\qquad \mathbf{N}=\mathbf{3 8 2}\end{array}$ & $\begin{array}{l}\text { Using Oral Hormone Therapy at } \\
\text { Baseline N=324 }\end{array}$ & P-value ${ }^{*}$ \\
\hline Change in DHEA (ng/ml) & $-0.17(1.06)$ & $-0.24(0.90)$ & 0.47 \\
\hline Baseline total E2 (pg/ml), median (IQR) & $8.5(8.0)$ & $17.6(14.8)$ & $<0.0001$ \\
\hline Change in total E2 (pg/ml) & $-5.4(26.7)$ & $-3.1(23.9)$ & 0.52 \\
\hline Baseline total $\mathrm{T}(\mathrm{pg} / \mathrm{ml}$ ), median (IQR) & $14.0(13.0)$ & $15.0(11.0)$ & 0.44 \\
\hline Change in total $\mathrm{T}(\mathrm{pg} / \mathrm{ml})$ & $-2.2(34.4)$ & $-4.1(51.3)$ & 0.59 \\
\hline Baseline LDL-cholesterol (mg/dl) & $137(36)$ & $120(29)$ & $<0.0001$ \\
\hline Change in LDL-cholesterol (mg/dl) & $-4(24)$ & $-1(22)$ & 0.24 \\
\hline Baseline HDL-cholesterol (mg/dl) & $48(11)$ & $55(14)$ & $<0.0001$ \\
\hline Change in HDL-cholesterol (mg/dl) & $1(7)$ & $1(8)$ & 0.36 \\
\hline Baseline triglycerides (mg/dl), median (IQR) & $154(90)$ & $181(87)$ & $<0.0001$ \\
\hline Change in triglycerides $(\mathrm{mg} / \mathrm{dl})$ & $-12(58)$ & $-10(86)$ & 0.81 \\
\hline
\end{tabular}

Abbreviations: $\mathrm{BMI}=$ body mass index; DHEA=dehydroepiandrosterone; $\mathrm{E} 2=$ =estradiol; HDL=high density lipoprotein; IQR=interquartile range; LDL=low density lipoprotein; SHBG=sex hormone binding globulin; T=testosterone

For sex hormones (FSH, SHBG, DHEA, total E2, T) at baseline where median (IQRs) are reported, Wilcoxon rank sum p-values reported. 


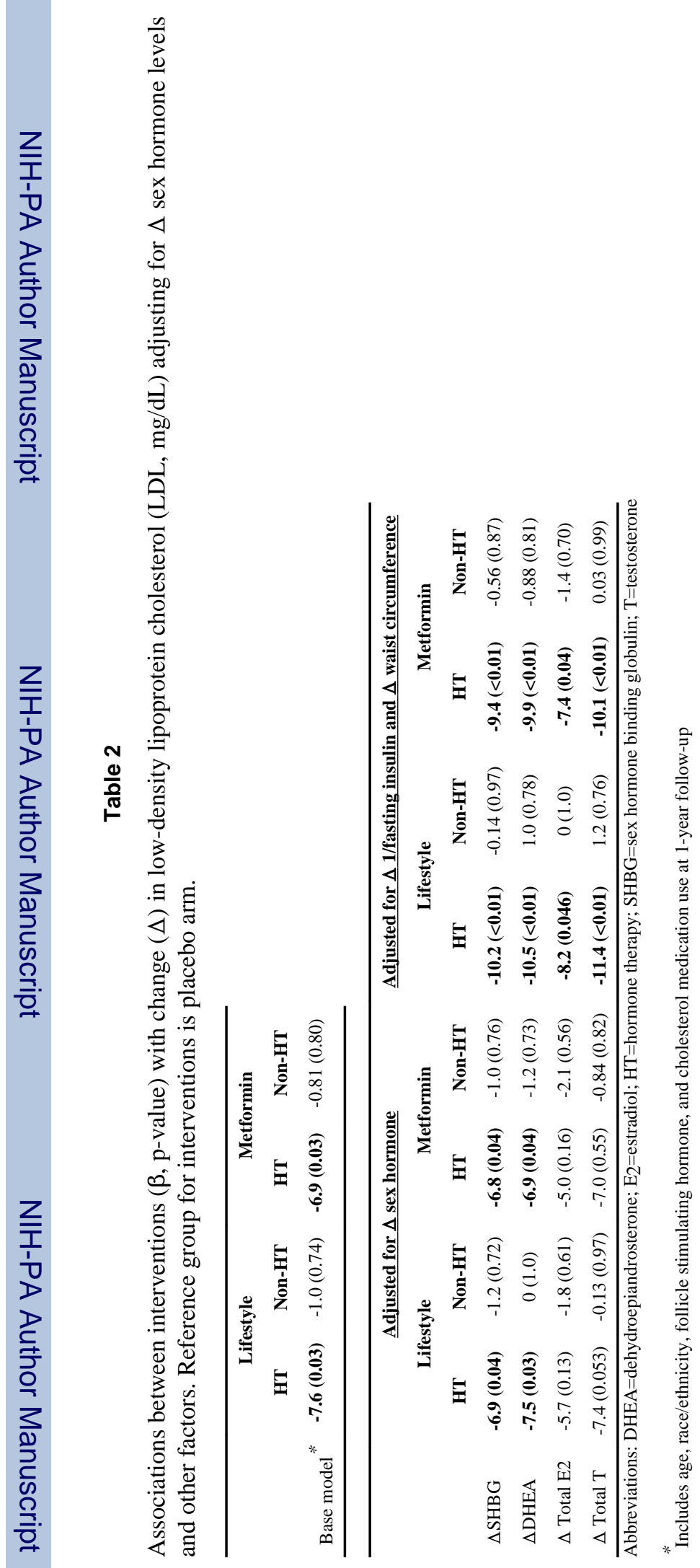

Metabolism. Author manuscript; available in PMC 2014 September 01. 

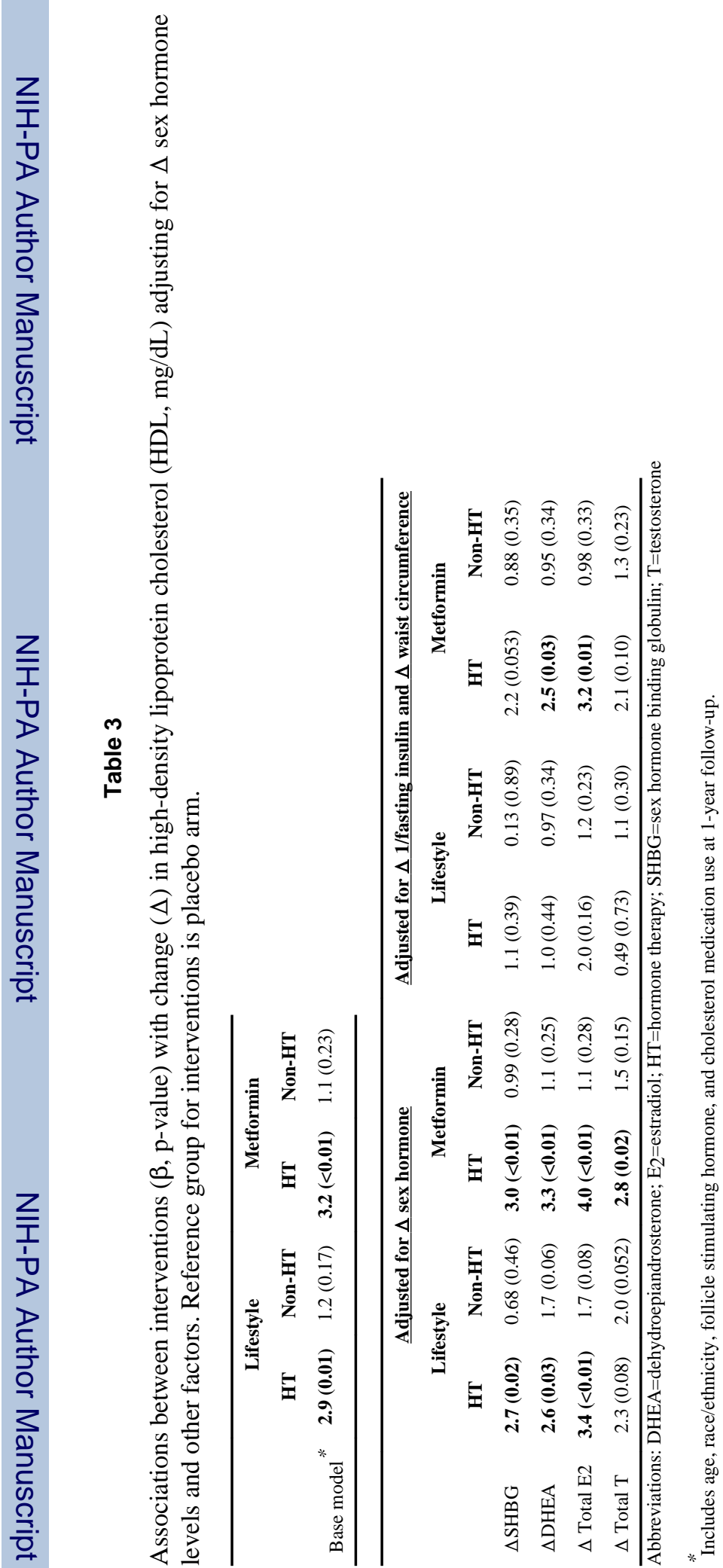

Metabolism. Author manuscript; available in PMC 2014 September 01. 


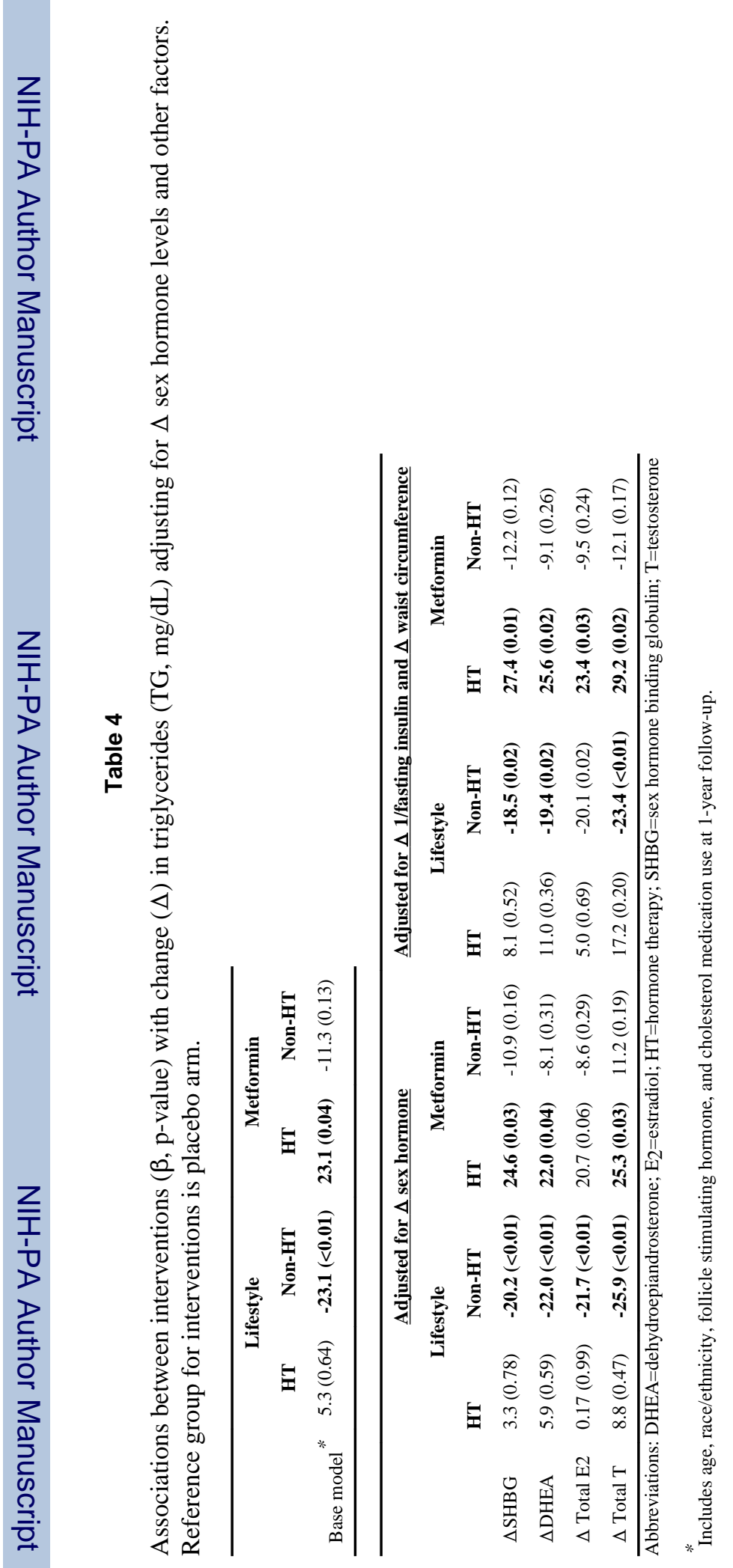

Metabolism. Author manuscript; available in PMC 2014 September 01. 